\title{
COMPOSIÇÃO NUTRICIONAL E CONTROLE DE QUALIDADE DO LEITE DE AMAPÁ DOCE (Brosimum parinarioides ducke)
}

\author{
ROSANA ANTUNES PALHETA* \\ RAIMUNDO FELIPE DA CRUZ FILHO* \\ ANA LUCIA BASÍLIO CARNEIRO*** \\ MARIA FRANCISCA SIMAS TEIXEIRA****
}

\begin{abstract}
Brosimum parinarioides, membro da família Moraceae, espécie vegetal nativa da região norte, conhecida como "amapazeiro", é fonte de látex, produto tradicionalmente consumido como alimento e usado na medicina popular por comunidades para o tratamento de patologia pulmonar e como tonificante. Embora o látex desse vegetal seja consumido como alimento funcional e tenha importância industrial farmacêutica, existem poucos estudos acerca da avaliação das propriedades dos compostos bioativos presentes nesse líquido. O objetivo deste trabalho foi avaliar a composição nutricional e analisar a qualidade do produto por dois métodos de extração do látex, visando orientar os agricultores extrativistas para aquisição de um alimento com qualidade para consumo humano. As amostras de látex foram submetidas à análise da composição centesimal, toxicidade e determinação da qualidade microbiológica. Os resultados demonstraram que o método de assepsia mais eficiente para a qualidade microbiológica do produto foi o uso da aspersão de hipoclorito de sódio seguido de álcool no caule da árvore. O látex apresentou compostos bioativos com propriedades antioxidantes, não apresentou efeito hemolítico em sangue de carneiro e nem toxicidade em relação à Artemia salina. Os resultados de composição centesimal demonstram que esse é um produto altamente energético, justificando seu uso como alimento matinal pelas populações da Amazônia.
\end{abstract}

PALAVRAS-CHAVE: SANITIZANTES; TOXICIDADE; CONTAMINAÇÃO DE ALIMENTO; FÍSICOQUÍMICA; Brosimum SP.

\footnotetext{
* Dra. Em Diversidade Biologia, Programa de Pós Graduação em Diversidade Biológica - Universidade Federal do Amazonas - UFAM (e-mail: rosanapalheta@ufam.edu.br)

** Dr. Em Biotecnologia, Programa de Pós Graduação em Biotecnologia - UFAM. Técnico do laboratório de microbiologia/UFAM (e-mail: rfi lho@ufam.edu.br).,

***Dra. Em biotecnologia, Profa. Professor adjunto UFPB, centro de Ciências da Saúde (CCS), Departamento de Morfologia.- 58051-900 João Pessoa - Paraíba - Brasil (e-mail: aluciabc@ig.com.br)

**** Profa. Dra. Orientadora do Programa de Pós Graduação em Diversidade Biológica, Curadora da Coleção de Culturas DPUA, Universidade Federal do Amazonas, Av. Gen. Rodrigo Octavio Jordão Ramos, 3000,

Campus Universitário, Manaus-AM. (e-mail: mteixeira@ufam.edu.br)
} 


\section{INTRODUÇÃO}

As plantas são um instrumento na promoção do bem-estar em muitas regiões do Brasil, país detentor de riquezas vegetais, tanto espécies como indivíduos, destacando-se inúmeros representantes com valor medicinal pelo significado que assumem na sociedade como um recurso biológico e cultural (AMARAL; SILVA, 2008).

Dentre os vegetais, a família Moraceae, representada por aproximadamente 50 gêneros e 1.500 espécies, tem em evidência o amapá ou amapá-doce - Brosimum potabile, $B$. utile e $B$. parinarioides. Dessas espécies, $B$. parinarioides possui um produto florestal não madeireiro de grande importância regional em função do valor medicinal e comestível do seu látex, líquido de sabor adocicado comumente consumido como leite de vaca, fazendo parte da alimentação em comunidades rurais na Amazônia (GALUPPO, 2004).

A árvore de Brosimum parinarioides atinge mais de 40 metros de altura, apresenta tronco cilíndrico e ereto, tem entrecasca avermelhada, com a madeira interna amarela, e ocorre nas Guianas e na bacia Amazônica. O látex dessa espécie tem aspecto viscoso, a coloração varia do branco a rosa claro e tem como constituintes proteínas, alcaloides, antraquinonas, derivados de cumarina, purinas, esteroides e triterpenoides (GALLUPO; PLOWDEN, 2005; FREITAS; FERNANDES, 2006). Além disso, têm destaque os teores de cálcio $(200,6 \mathrm{mg} / \mathrm{kg})$; fósforo $(149,53 \mathrm{mg} / \mathrm{kg})$ e potássio $(1.862,06 \mathrm{mg} / \mathrm{kg}$ ) em relação aos demais minerais (MATTIETTO et al., 2009).

Assim, a ausência de cuidados assépticos na manipulação do látex de Brosimum parinarioides proporciona redução da qualidade e da vida útil durante o armazenamento, tornando esse produto não madeireiro impróprio para consumo em consequência do aumento da carga microbiana. Portanto, a comercialização do produto alimentício em desacordo com padrões de qualidade microbiológica poderá veicular espécies de microrganismos patogênicos ao consumidor, e adotar medidas de controle a fim de prevenir, reduzir a um nível aceitável ou eliminar o agente contaminante proporciona a melhoria da qualidade do produto, chave para o sucesso em qualquer ramo de atividade (ETO et al., 2010; ELPO et al., 2004).

O objetivo deste trabalho foi analisar a qualidade do produto obtido por dois métodos de extração do látex e avaliar a composição nutricional, visando orientar os agricultores extrativistas para aquisição de um alimento com qualidade para consumo humano.

\section{MATERIAL E MÉTODOS}

\subsection{EXTRAÇÃO DO LÁTEX}

As amostras de látex foram coletadas de árvores selecionadas na floresta do Campus Manaus da Universidade Federal do Amazonas (UFAM). Para retirada do látex, no caule de cada árvore, a uma altura de $100 \mathrm{~cm}$ do solo, foi delimitada uma área retangular medindo $50 \times 100 \mathrm{~cm}$. Em cada caule demarcado foram realizados os seguintes tratamentos: Tratamento 1, aspersão de álcool etílico $70 \%$ seguida de aspersão de água destilada esterilizada a $121^{\circ} \mathrm{C}$ por 45 minutos; Tratamento 2 aspersão de hipoclorito de sódio $2 \%(\mathrm{v} / \mathrm{v})$; Tratamento 3: aplicação do hipoclorito de sódio $2 \%(\mathrm{v} / \mathrm{v})$ seguido de aspersão de álcool etílico $70 \%$, seguindo-se a recomendação de Santos et al. (1998). As coletas foram realizadas sempre nas primeiras horas do dia.

De cada árvore foram coletadas três amostras, cada uma correspondendo a $50 \mathrm{~mL}$ de látex in natura, totalizando doze repetições. O produto foi envasado em frascos de Erlenmeyer de $125 \mathrm{~mL}$, esterilizados a $121^{\circ} \mathrm{C}$ por 45 minutos. Todos os recipientes foram mantidos sob refrigeração e transportados para o laboratório da Coleção de Cultura DPUA/UFAM. O tempo percorrido entre as análises e o processamento das amostras não ultrapassou 4 horas. Das amostras coletadas foram analisadas: qualidade microbiológica, toxicidade em relação à Artemia salina, atividade de hemólise, atividade antioxidante e análise físico-química. 
Nas análises microbiológicas, em condições assépticas, $10 \mathrm{~mL}$ de cada amostra foram transferidos para $90 \mathrm{~mL}$ de água peptonada $0,1 \%$ esterilizada, em frasco de Erlenmeyer de 500 $\mathrm{mL}$ (diluição $10^{-1}$ ). A partir dessa diluição foram preparadas diluições sucessivas até obtenção da diluição $10^{-3}$ utilizando-se $9 \mathrm{~mL}$ do mesmo diluente (SILVA et al., 2007b). Todos os experimentos foram realizados em triplicata.

\subsection{CONTAGEM TOTAL DE AERÓBIOS MESÓFILOS EM PLACAS}

As determinações de microrganismos viáveis totais (mesófilos, fungos filamentosos e leveduras) foram realizadas pelo método de contagem em placas (Silva et al., 2007b). Para o isolamento de aeróbios mesófilos, de cada uma das diluições, o volume de $100 \mu \mathrm{L}$ foi semeado na superfície do Ágar PCA (ágar padrão para contagem). O cultivo foi realizado por espalhamento com o auxílio de alça de Drigalski até completa absorção da suspensão do látex seguindo-se a incubação $\left(37^{\circ} \mathrm{C} / 24-48 \mathrm{~h}\right)$. Após incubação realizou-se a contagem das colônias.

Para quantificação foi calculada a média das contagens de colônias multiplicada pelo inverso da diluição, expressando-se o quantitativo de unidades formadoras de colônia por mL (UFC/ $\mathrm{mL}$ ) de produto.

\subsection{CONTAGEM DE COLIFORMES TOTAIS, COLIFORMES TERMOTOLERANTES}

A prova presuntiva foi realizada utilizando-se a técnica dos tubos múltiplos contendo tubos de Durham invertidos, o volume de $1.000 \mu \mathrm{L}$ de cada série de diluições $\left(10^{-1}, 10^{-2}\right.$ e $\left.10^{-3}\right)$ foi inoculado em tubos contendo Caldo Lauril Sulfato Triptose (LST) e caldo Escherichia coli (EC) em triplicata, os quais foram incubados a $35^{\circ} \mathrm{C}$ e a $45^{\circ} \mathrm{C}$ por 24 a 48 horas respectivamente, observando-se a formação de gás no interior do tubo. $O$ cálculo do Número Mais Provável (NMP) foi efetuado segundo a citação de Silva et al., 2007b.

\subsection{ISOLAMENTO DE FUNGOS FILAMENTOSOS E LEVEDURAS}

Após completa homogeneização de cada suspensão celular foi retirada alíquota equivalente a $200 \mu \mathrm{L}$ para inoculação na superfície do Ágar Rosa Bengala Cloranfenicol (RBC), seguido por espalhamento com alça de Drigalski até completa absorção da suspensão celular. As placas invertidas foram mantidas a $25^{\circ} \mathrm{C}$ durante sete dias, observando-se o crescimento dos microrganismos a cada 24 horas. Todos os experimentos foram realizados em duplicata. Para quantificação foi calculada a média das contagens de colônias multiplicada pelo inverso da diluição, expressando-se o quantitativo de UFC/mL de produto.

De acordo com as características macroscópicas, as colônias semelhantes foram repicadas para tubos de ensaio contendo meios específicos para gênero, seguindo a identificação ao nível de espécie.

Os fungos filamentosos foram identificados com base nas estruturas de reprodução, características macro e micromorfológicas nos cultivos em meio sólido seletivo (Czapeck, Batata dextrose - BDA, Extrato de Malte e Czapek Yeast Extract Ágar - CYA), seguindo-se as recomendações de Pitt (1985) e Samson et al. (1995).

\subsection{MÉTODOS ANALÍTICOS}

\subsubsection{Obtenção do Extrato}

Para os testes de atividade antioxidante e atividade hemolítica, o látex foi primeiramente desidratado a $45^{\circ} \mathrm{C}$ e posteriormente submetido à extração com os solventes metanol e hexano PA, a $25^{\circ} \mathrm{C}$ por 24 horas, realizando-se em seguida filtração e evaporação do solvente sob pressão. 
Para as análises, o extrato foi ressuspendido em solução aquosa contendo $10 \%$ de Dimetilsulfóxido (DMSO) na concentração desejada para cada um dos testes posteriores.

\subsubsection{Atividade de Hemólise}

A atividade de hemólise in vitro foi realizada segundo a metodologia de Oliveira et al. (2009). Para este experimento utilizou-se uma solução a 1\% (v/v) de células de hemácias do sangue comercial de carneiro em tampão fosfato $0,1 \mathrm{M} \mathrm{pH} \mathrm{7,4.} \mathrm{O} \mathrm{extrato} \mathrm{foi} \mathrm{testado} \mathrm{em} \mathrm{concentrações}$ de $500,250,100$ e $50 \mathrm{mg} / \mathrm{mL}$. A atividade hemolítica foi avaliada por comparação com o efeito de um controle positivo (saponina comercial a $10 \%$ agente hemolítico) e controle negativo (solução tampão). O resultado da ação hemolítica do extrato foi interpretado qualitativamente, observando-se a coloração da solução após 24 horas de contato com o extrato. Todas as análises foram realizadas em triplicata.

\subsubsection{Atividade Antioxidante - Quantificação de Fenóis Totais}

A quantificação de fenóis totais foi realizada por meio de espectroscopia na região do visível pelo método de Folin-Dennis. O teor de fenóis totais presentes nos extratos metanol e hexano foi determinado por interpolação da absorbância das amostras contra uma curva de calibração construída com padrões de ácido tânico (10 a $1000 \mu \mathrm{g} / \mathrm{mL})$ a $720 \mathrm{~nm}$. A equação da curva de calibração foi $\boldsymbol{y}=\mathbf{0 , 0 3 2 5} \boldsymbol{x}-\mathbf{0 , 0 3 3 4}$ e o coeficiente de correlação $R=0,9553$.

\subsubsection{Bioensaio de Toxicidade em Artemia Salina}

Para eclosão dos cistos de $A$. salina utilizou-se solução de sal marinho 3\% (p/v) esterilizada a $121^{\circ} \mathrm{C}$ por 15 minutos, $\mathrm{pH} 6,5$. O quantitativo de $0,1 \mathrm{~g}$ de cistos do microcrustáceo foi adicionado nessa solução e incubado durante 30 horas, a $30^{\circ} \mathrm{C}$, sob luminosidade (40 watts) e agitação constante (140 rpm), conforme o método de Harwig e Scott (1971) modificado por Atayde (2008).

Após eclosão dos cistos, $100 \mu \mathrm{L}$ da solução contendo náuplios foi transferido para poços individuais de placas multipoços $(6 \times 4)$, seguindo-se a inoculação do extrato enzimático em diferentes concentrações $(1.000 \mathrm{mg} / \mathrm{mL}$ a $100 \mathrm{mg} / \mathrm{L})$ completando-se o volume final de cada poço para $1 \mathrm{~mL}$ com solução salina sem larvas. Após 16 horas de contato com o extrato enzimático e com o branco foi determinada a taxa de mortalidade segundo a classificação de Harwig e Scott (1971), conforme a seguinte expressão:

mortalidade $=$ mortalidade dos individuos mortos $\times 100$ número total de indivíduos

O grau de toxicidade (mortalidade \%) foi classificado segundo Harwig e Scott (1971): 0 $-9 \%$ = não tóxico (NT); $10-49 \%$ = ligeiramente tóxico (LT); $50-89 \%=$ tóxico (T); $90-100 \%=$ altamente tóxico (AT).

\subsubsection{Caracterização Físico-Química do Látex}

Amostras foram separadas, em triplicata, aleatoriamente, para a determinação do $\mathrm{pH}$ e da acidez titulável, conforme as normas analíticas do Instituto Adolfo Lutz (2008).

A análise dos teores de umidade, cinzas, proteínas, lipídios e fibras foi realizada em triplicata, de acordo com as normas analíticas do Instituto Adolfo Lutz (2008). O teor de carboidratos totais, diminuindo do valor 100 o somatório de proteínas, lipídios, cinzas e umidade, representado pela fração NIFEXT (livre de nitrogênio), foi calculado por diferença em relação às demais frações e o valor energético a partir do fator de conversão de Atwater centesimal aproximada, utilizando-se os seguintes fatores clássicos de conversão de Atwater: 9 kcal por grama de lipídios, 4 kcal por grama de proteínas e 4 kcal por grama de carboidratos. 


\section{RESULTADOS E DISCUSSÃO}

Das amostras de látex extraídas do caule de Brosimum parinarioides submetidas a três diferentes tratamentos pode-se observar que nos tratamentos 1 e 2 foram isoladas em média $7,56 \mathrm{x}$ $10^{5}$ e $9,0 \times 10^{5}$ UFC de fungos filamentosos, respectivamente. A frequência de colônia no látex extraído após o tratamento 1 foi $99 \%$ superior ao quantitativo de microrganismos registrado após o tratamento 2. Todavia, quando aplicado o tratamento 3 , não foi detectada a presença de microrganismo. A partir dos dados obtidos foram identificados somente fungos filamentosos anamórficos (7,53 x 10 $\mathrm{UFC} /$ $\mathrm{mL}$ ) e ascomiceto $\left(3,0 \times 10^{3} \mathrm{UFC} / \mathrm{mL}\right)$, não se registrando ocorrência de bactérias (Tabela 1). Dessa forma, a desinfecção realizada separadamente com álcool $70 \%$ (tratamento 1) ou hipoclorito de sódio $2 \%$ (tratamento 2 ) foi ineficaz para a higienização do caule da árvore de $B$. parinarioides no processo de extração do látex. $\mathrm{O}$ valor do $\mathrm{pH}$ do látex foi em torno de 5,5 (ligeiramente ácido), não havendo diferença significativa entre as amostras.

\section{TABELA 1. FREQUÊNCIA DE UFC DOS MICRORGANISMOS ISOLADOS DO LÁTEX DE BROSIMUM PARINARIOIDES COLETADO SOB DIFERENTES TRATAMENTOS DE DESINFECÇÃO (UFC/ML)}

\begin{tabular}{lccc}
\hline \multirow{2}{*}{ Microrganismos } & \multicolumn{2}{c}{ Tratamentos de desinfecção (T) } \\
\cline { 2 - 4 } Fungos filamentosos & T1 & T2 & T3 \\
\hline Fungos Anamorfos & $7,56 \times 10^{5}$ & 90 & ND \\
\hline Ascomicetos & $7,53 \times 10^{3}$ & 75 & ND \\
\hline Bactérias aeróbias Mesófilas & $3,00 \times 10^{3}$ & 15 & ND \\
\hline Coliformes totais & ND & ND & ND \\
\hline
\end{tabular}

ND - Não detectado; T1 - álcool 70\%; T2 - Hipoclorito de sódio; T3 - Hipoclorito de sódio seguido de álcool 70\%

Em consideração ao quantitativo de UFC de fungos filamentosos isolados, provavelmente a baixa frequência esteja relacionada à sensibilidade do método utilizado e do quantitativo de microrganismos presentes na época da coleta. Quanto à ausência do isolamento de bactérias, pode-se inferir que o $\mathrm{pH}$ do látex seja um fator limitante para o crescimento desses microrganismos, uma vez que se desenvolvem em pH próximo da neutralidade, enquanto os fungos se desenvolvem em pH 5,6 (DEACON, 2009; GAVA et al., 2009).

Quanto ao efeito das substâncias utilizadas na desinfecção do caule, Silva et al. (2007a) citam que o hipoclorito de sódio oxida os grupos hidroxila de enzimas e tem ação direta nos grupos aminados de proteínas celulares, o que promove a morte de microrganismos. Além disso, o pH 11,0 dessa solução proporciona meio desfavorável para o desenvolvimento de bactérias e fungos. $O$ álcool etílico causa desnaturação das proteínas quando na presença de água e remoção de lipídios, e também exibe atividade reduzida contra certos fungos e vírus, embora não seja útil à esterilização por não apresentar atividade contra esporos bacterianos (KALIL; COSTA, 1994; SANTOS et al., 2002).

Em relação aos fungos filamentosos encontrados no produto após os tratamentos 1 e 2 , determinou-se a ocorrência de 10 espécies distribuídas em 4 gêneros, dos quais 9 são fungos anamorfos e somente um do filo Ascomycota (Tabela 2). O gênero Penicillium foi o que apresentou o maior número de espécies - $6(33,5 \%)$-, representadas por $P$. citreonigrum $(23,807 \%), P$. felutanum $(0,001 \%)$, P. janckzenwiskii $(9,261 \%)$, P. janthinellum $(0,265 \%)$, P. lividum $(0,001 \%)$ e P. restrictum $(0,132 \%)$. Os demais fungos encontrados neste trabalho foram Aspergillus $(0,001 \%)$, 
Fusarium (13,23\%), Verticillium (52,904\%) e Eurotium (0,399\%) com ocorrência de apenas uma espécie para cada gênero (Tabela 2). Destes, as espécies de maior ocorrência nesta investigação foram V. fungicola (53\%), P. citreonigum (23,807\%) e F. sporotrichioides (13\%). Das três espécies de fungos filamentosos de maior ocorrência, duas tem sido citadas como espécies produtoras de micotoxinas, uma associada ao beriberi ( $P$. citreonigum) e a outra ( $F$. sporotrichioides) causadora de adenomas pulmonares e hepáticos em ratos. A presença de fungos em material vegetal de uso medicinal pode ser prejudicial predominantemente a pacientes imunodeprimidos, uma vez que podem causar alergias ou outras patologias quando inalados, ingeridos ou inoculados (CARMO et al., 2007; SOUZA et al., 2006).

\section{TABELA 2. FREQUÊNCIA DE UFC/ML E FREQUÊNCIA DAS ESPÉCIES DE FUNGOS ISOLADOS DO LÁTEX DE BROSIMUM PARINARIOIDES COLETADO SOB DIFERENTES CONDIÇÕES DE DESINFECÇÃO}

\begin{tabular}{|c|c|c|c|c|}
\hline \multirow{2}{*}{ Espécies identificadas } & \multicolumn{3}{|c|}{$\mathrm{UFC} / \mathrm{mL}$} & \multirow{2}{*}{$\begin{array}{c}\text { Porcentagem } \\
\text { total (\%) }\end{array}$} \\
\hline & T1 & T2 & T3 & \\
\hline \multicolumn{5}{|l|}{ Fungos Anamorfos } \\
\hline Aspergillus candidus & ausente & 5 & ausente & 0,001 \\
\hline Fusarium sporotrichioides & $10,0 \times 10^{4}$ & 30 & ausente & 13,230 \\
\hline Penicillium citreonigrum & $18,0 \times 10^{4}$ & ausente & ausente & 23,807 \\
\hline Penicillium felutanum & ausente & 5 & ausente & 0,001 \\
\hline Penicillium janckzenwiskii & $7,0 \times 10^{4}$ & 20 & ausente & 9,261 \\
\hline Penicillium janthinelum & $0,2 \times 10^{4}$ & 5 & ausente & 0,265 \\
\hline Penicillium lividum & ausente & 5 & ausente & 0,001 \\
\hline Penicillium restrictum & $0,1 \times 10^{4}$ & $\mathrm{Nd}$ & ausente & 0,132 \\
\hline Verticilium fungicola & $40,0 \times 10^{4}$ & 5 & ausente & 52,904 \\
\hline \multicolumn{5}{|l|}{ Filo Ascomycota } \\
\hline Eurotium amstelodami & $0,3 \times 10^{4}$ & 15 & ausente & 0,399 \\
\hline TOTAL (UFC) & $75,6 \times 10^{4}$ & 90 & ausente & \\
\hline FREQUÊNCIA TOTAL (\%) & 99,988 & 0,012 & ausente & 100 \\
\hline
\end{tabular}

T1 - álcool 70\%; T2 - Hipoclorito de sódio; T3 - Hipoclorito de sódio seguido de álcool 70\%

Os resultados dos ensaios de toxicidade contra Artemia salina e os testes de atividade de hemólise mostraram que o extrato obtido do látex não apresentou toxicidade segundo a classificação de HARWIG e SCOTT (1971), pois não houve mortalidade dos microcrustáceos nem atividade hemolítica nas concentrações testadas utilizando sangue comercial de carneiro.

Com base nesses dados, o látex de $B$. parinarioides, um alimento não convencional, é relativamente seguro na forma de uso pelo povo nativo da Amazônia. O bioensaio com Artemia continua sendo uma forma útil e economicamente viável para avaliação de extratos originários da biodiversidade microbiana e de vegetais, todavia não é adequado para elucidação dos mecanismos de ação dos extratos dos seus constituintes (FAVILLA et al., 2006).

Em relação à atividade antioxidante, diversas pesquisas têm relacionado o teor de fenóis totais, como taninos e flavonoides, com a atividade biológica (antioxidante e antimicrobiana). Assim, 
a partir da curva de calibração, observou-se que esses compostos bioativos estão presentes nos extratos obtidos a partir do metanol $(430 \mu \mathrm{g})$ em equivalente ácido tânico; já no extrato hexânico, os mesmos não foram detectados. Diversos autores afirmam que as propriedades benéficas desses antioxidantes estão relacionadas à sua capacidade de sequestrar os radicais livres, as moléculas responsáveis pelo envelhecimento nos seres humanos (SOUZA et al., 2007; ANGELO; JORGE, 2007; FUNARI; FERRO, 2006).

A composição centesimal $(\mathrm{g} / 100 \mathrm{~g})$ do látex de $B$. parinarioides citada na Tabela 3 mostrou que o produto tem alto conteúdo de lipídios $(34,64)$ e energia $(343,2 \mathrm{Kcal})$, baixo teor de proteína $(2,14)$, carboidrato $(5,72)$, cinzas $(2,51)$ e pH ligeiramente ácido.

Essas características mostram que o látex é um excelente energético, o que provavelmente justifica o seu uso como alimento quando adicionado ao leite no café da manhã pela população amazônica da região norte do Brasil.

TABELA 3. COMPOSIÇÃO FíSICO-QUÍMICA DO LÁTEX DE BROSIMUM PARINARIOIDES (G/100G).

\begin{tabular}{ccccccc}
\hline Umidade & Lipídios & Proteína & Cinzas & Carboidratos & Fibra & Energia (Kcal) \\
\hline 54,99 & 34,64 & 2,14 & 2,51 & 5,72 & 2,04 & 343,2 \\
\hline
\end{tabular}

\section{CONCLUSÃO}

Os resultados evidenciaram a eficácia dos procedimentos higiênico-sanitários ao se utilizar o tratamento por aspersão dos agentes sanitizantes (hipoclorito de sódio seguido de álcool) na coleta do látex de B. parinarioides, tecnologia que pode ser aplicada na cadeia produtiva. Além disso, a determinação da composição centesimal indicou que se trata de um produto com alto valor energético, propriedades antioxidantes e ausência de toxicidade.

\section{ABSTRACT}

\section{NUTRITIONAL COMPOSITION AND QUALITY CONTROL OF SWEET AMAPÁ MILK (Brosimum parinarioides Ducke)}

Brosimum parinarioides is a plant species member of the Moraceae family, native of the northern region, known as "amapazeiro". It is a source of latex traditionally consumed as food and used in folk medicine to treat pulmonary pathologies and as an invigorative. Although the latex from this plant is consumed as functional food and has pharmaceutical industrial importance, few studies evaluated the properties of the bioactive compounds present in this liquid. The aim of this study was to evaluate the nutritional composition and analyze the quality of the product by two latex extracting methods to guide farmers to extract quality food for human consumption. The latex samples were analyzed for chemical composition, toxicity and determination of microbiological quality. The results showed that the most efficient sterilization method for microbiological quality was the use of spray sodium hypochlorite followed by alcohol in the tree stem. The latex showed bioactive compounds with antioxidant properties, no hemolytic effect on sheep blood, nor toxicity regarding Artemia salina. The results of the chemical composition show that this is a highly energetic product, justifying its use as morning food by Amazonian populations. 


\section{REFERÊNCIAS}

1 AMARAL, E. A.; SILVA, R. M. G. Avaliação da toxidade aguda de angico (Anadenanthera falcata), pau-santo (Kilmeyera coreacea), aroeira (Myracrodruon urundeuva) e cipó-de-são-joão (Pyrostegia venusta), por meio do bioensaio com Artemia salina. Perquirere (UNIPAM. Online), v. 5, p. 01-16, 2008.

2 ANGELO, P.M., JORGE N. Compostos fenólicos em alimentos - Uma breve revisão. Revista Instituto Adolfo Lutz, v. 66, n. 1, p. 232-240, 2007.

3 ATAYDE, H.M. Potencial de toxicidade de microfungos isolados de rações para peixes fabricadas no estado do Amazonas. 2008. 80 p. Dissertação (Mestrado e, Ciência dos alimentos - Universidade Federal do Amazonas, Manaus, 2008.

4 CARMO, E. S.; BELÉM, L. F.; CATÃO, R. M. R.; LIMA, E. O.; SILVEIRA, I. L.; SOARES, L. H. M. Microbiota fúngica presente em diversos setores de um hospital público em Campina Grande - PB. RBAC. v. 39, n. 3, p. 213-216. 2007.

5 DEACON, J.W. Fungal Biology. Third edition. Oxford: Blackwell Science Ltd; 2009. 384p.

6 ELPO, E. R. S.; NEGRELLE, R. R. B.; GOMES, E. C. Avaliação da qualidade microbiológica do gengibre "in natura" comercializado na região metropolitana de Curitiba, PR. Visão Acadêmica, Curitiba, v. 5, n. 2, p. 139 -146, Jul.- Dez. 2004.

7 ETO, D. K; KANO, A. M; BORGES, M. T. M. R.; BRUGNARO, C.; CECCATO-ANTONINI, S. R.; VERRUMA-BERNARDI, M. R. Qualidade microbiológica e físico-química da polpa e mix de açaí armazenada sob congelamento. Revista Instituto Adolfo Lutz. v. 69, n. 3, p. 304-310, 2010.

8 FAVILLA, M; MACCHIA, L; GALLO, A.; ALTOMARE, C. Toxicity assessment of metabolites of fungal biocontrol agent using two diferent (Artemia salina and Daphnia magna) invertebrate boassays. Food and Chemical Toxicology. v. 44, p. 1922-1931, 2006.

9 FREITAS, J.C., FERNANDES MEB. Uso de plantas medicinais pela comunidade de Enfarrusca. Boletim Museu Paraense Emílio Goeldi. Ciências Naturais, v. 1, p. 11-26, 2006.

10 FUNARI, C., FERRO, V.O. Análise de Própolis. Ciência Tecnologia Alimentos; v. 26, n. 1, p. 171-178. 2006

11 GALLUPO, S., PLOWDEN, C. Amapá: o fortificante da Amazônia. In: SHANLEY, P (org.) Frutíferas e Plantas Úteis na Vida Amazônica. $1^{a}$ Edição. Belém: CIFOR; 2005. v.1 p.1-101.

12 GALUPPO, S. C., Documentação do uso e valorização do óleo de piquiá (Caryocar viillllosum) e do leite do amapá--doce (Brosimum parinarioides) para a comunidade de Piquiatuba, floresta nacional do tapajós. Estudos físicos, químicos, fitoquímicos e farmacológicos. 2004. 92. Dissertação - Universidade Federal Rural da Amazônia, Belém. 2004.

13 GAVA, A. J, SILVA, C. A. B.; FRIAS, J.R.G Tecnologia de Alimentos: princípios e aplicações. São Paulo: Nobel, 2009. 664p.

14 HARWIG J, SCOTT PM. Brine Shrimp (Artemia salina L.) Larvae as a Screening System for Fungal Toxins. Applied Microbiology. v. 21, p.1011-1016, 1971.

15 INSTITUTO ADOLFO LUTZ (2008). Instituto Adolfo Lutz (São Paulo). Métodos físico-químicos para análise de alimentos/ coordenadores Odair Zenebon, Neus Sadocco Pascuet e Paulo Tiglea -- São Paulo: Instituto Adolfo Lutz, 2008.

16 KALIL EM, COSTA AJF. Desinfecção e esterilização. Acta Ortopédica Brasileira. v. 2 p.1-4, 1994.

17 MATTIETTO, R. A. ; BEZERRA, V.S. ; TAXI, C. D. ; YANO, C. Y. B. ; CORDEIRO, B.S.; TSUKUI, A. . Otimização do processo de conservação do leite de amapá-doce (Brosimum parinarioides Ducke). Anais In: Conferência do Subprograma de Ciência e Tecnologia SPC\&T Fase II/PPG7, 2009, Belém. Conferência do Subprograma de Ciência e Tecnologia SPC\&T Fase II/PPG7. Brasília : CNPq, 2009. p. 352-354. http://www.alice.cnptia.embrapa.br/handle/ doc/877765

18 OLIVEIRA VMA, CARNEIRO ALB, CAUPER GSB, POHLIT AM. In vitro screening of Amazonian plants for hemolytic activity and inhibition of platelet aggregation in human blood. Acta Amazonica. v. 39, n. 4, p. $973-980,2009$

19 PITT, J.I. A laboratory guide to common Penicillium species. 1st Edition North Ryde: Commonwealth Scientific and Industrial Research Organization; 1985.

20 SAMSON, R.A., HOEKSTRA, S.E, FRISVAD, J.C., FILTENBORG, O. Introduction to Food Borne Fungi. Baar: Centralalbureau voor Schimmelcultures. 1995 p. 299.

21 SANTOS, A.A.M., VEROTTI, M.P., SANMARTIN, J.Á, MESIANO, E.R.A.B. Importância do álcool no controle de infecções em serviços de saúde. RAS 2002; v 4, p. 7-14 disponível em: http://www7.anvisa.gov.br/servicosaude/controle/controle_ alcool.pdf. Acesso em 12/07/2011.

22 SANTOS, I.M., VEN NCIO, A., LIMA, N. Fungos contaminantes na indústria alimentar. $1^{a}$ Edição. Braga: Micoteca da Universidade do Minho - Centro de Engenharia Biológica; 1998. p. 128.

23 SILVA, L.A.F.; COELHO, K.O; DAMASCENO, A.D.; NICOLAU, E.S.; ANDRADE, M.A.; FIORAVANTI, M.C.S.; MESQUITA, A.J.; BARBOSA, V.T.; MOURA, M.I. Avaliação da concentração e do efeito sanitizante do hipoclorito de sódio em pedilúvio para bovinos. Semina: Ciências Agrárias. v. 28, p. 89-96. 2007a.

24 SILVA, N., JUNQUEIRA, V. C. A., SILVEIRA, N. F.A. Manual de métodos de análise microbiológica de alimentos. $4^{a}$ edição. São Paulo: Livraria Varela. 2007b.

25 SOUZA, C.M.M.; SILVA, H.R, VIEIRA-JR, G.M., AYRES, M.C.C., COSTA, C.L.L., CAVALCANTE, L. C. D; BARROS, E. D. S.; ARAÚJO, P. B. M. , BRANDÃO, M. S., CHAVES, M. H. Fenóis totais e atividade antioxidante de cinco plantas medicinais. Quimica Nova, v. 30, n. 2, p. 351-355, 2007.

26 SOUZA, T.P., LIONZO, M. I., PETROVICK, P. R. Avaliação da redução da carga microbiana de droga, vegetal através do processamento tecnológico: decocção e secagem por aspersão. Revista Brasileira Farmacognosia. v.16, p. 94-98, 2006. 